NOTE

\title{
Lactococcus garvieae in wild Red Sea wrasse Coris aygula (Labridae)
}

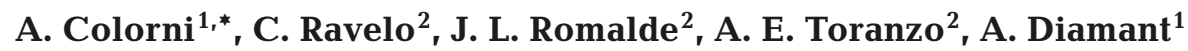 \\ ${ }^{1}$ Israel Oceanographic and Limnological Research, National Center for Mariculture, PO Box 1212, Eilat 88112, Israel \\ ${ }^{2}$ Departamento de Microbiología y Parasitología, Facultad de Biología, Universidad de Santiago de Compostela, \\ 15782 Santiago de Compostela, Spain
}

\begin{abstract}
Lactococcus garvieae infection in wild wrasse Coris aygula is reported, and the serological and molecular characteristics of the isolate are described. This is the first evidence of the presence of this pathogen in the Red Sea, and it follows the recent diagnosis of Mycobacterium marinum and Streptococcus iniae in wild fish from the same region. Whether all 3 pathogens are strains endemic to the Red Sea, or recent introductions into the region, remains to be determined, but their appearance over a period of a few years in wild fish populations in the northern Red Sea is consistent with an emerging trend affecting marine organisms on a global level in areas subjected to intense anthropogenic impacts.
\end{abstract}

KEY WORDS: Lactococcus garvieae - Wild · Wrasse $\cdot$ Red Sea $\cdot$ Israel $\cdot$ Clindamycin

Resale or republication not permitted without written consent of the publisher

A case of Lactococcus garvieae infection in a wild wrasse Coris aygula Lacepède 1801 (Labridae) was diagnosed in the Gulf of Eilat (Red Sea, Israel) in November 2002. An adult male $(610 \mathrm{~mm}$ total length [TL], weighing $3390 \mathrm{~g}$ ) was collected by divers who found it lying motionless in shallow (4 to $5 \mathrm{~m}$ ) water at the southern edge of the Eilat Nature Reserve. Fishing and boating are forbidden in the area, and no superficial lesions were evident on the fish to indicate a capture attempt or any traumatic impact. C. aygula is a fairly common species in the Gulf of Eilat, with a wide distribution in the Indo-Pacific. The adult is a solitary, reef-inhabiting fish that feeds chiefly on shelled molluscs, hermit crabs and sea urchins (Randall 1983). Physical parameters in the Gulf of Eilat are subject to limited seasonal fluctuations; water temperature was approximately $25^{\circ} \mathrm{C}$, and salinity in this region is fairly constant at $40 \mathrm{ppt}$.

The fish was taken to the National Center for Mariculture for examination and subjected to thorough gross-pathological, parasitological, histopathological and bacteriological investigation. A moderate infection of Gnathia sp. (Isopoda) pranizae, both with and without engorged guts, was observed on the gills. A few nematodes and tetraphyllid cestodes were found in the gut lumen. Samples of spleen, kidney, liver, intestine and brain were fixed in buffered neutral formalin, embedded in paraffin blocks and cut at $6 \mu \mathrm{m}$ thickness, according to standard procedures (Sheehan \& Hrapchak 1980). Histological sections were stained with hematoxylin and eosin (H\&E) and Gram stains. In the sections, a few larval nematodes were found encysted in the gut wall. Liver and kidney displayed foci of ceroid aggregates. However, apart from some hemorrhage in the kidney, spleen and liver, a systemic presence of Gram-positive cocci and early signs of tissue autolysis, no pathological lesions were visible. Blood and spleen samples were inoculated on tryptic soy agar (TSA) prepared with $25 \%$ aged and filtered seawater and supplemented with $5 \%$ outdated human blood-bank blood. Pure cultures of $\alpha$-hemolytic Grampositive cocci in chains, negative for oxidase and catalase tests, were obtained. Presumptive identification of the isolate was made using the Rapid ID 32 Strep, API 20 Strep and API 50-CH miniaturized kits (bioMérieux, Marcy l'Etoile), according to the recommendations of Ravelo et al. (2001a). Manufacturer's instructions were followed, except for the incubation temperature, maintained at $24 \pm 1^{\circ} \mathrm{C}$ instead of the recommended $36 \pm$ $1^{\circ} \mathrm{C}$, and the intermediate readings, which were not taken before $18 \mathrm{~h}$. Final results were read after $72 \mathrm{~h}$ incubation.

The biochemical profile of the isolate, according to the API 20 Strep and API 50-CH database, matched the identification of Lactococcus lactis ssp. lactis, which is known to be nearly indistinguishable bio- 
chemically from L. garvieae (Collins et al. 1983, Elliott \& Facklam 1996, Teixeira et al. 1996), and which, in turn, is a synonym of Enterococcus seriolicida (Eldar et al. 1996, Teixeira et al. 1996). The profile obtained with Rapid ID 32 Strep (30333101111) corresponded with L. garvieae (99.6\% confidence). Resistance to clindamycin, which according to Elliott \& Facklam (1996) should differentiate L. garvieae from L. lactis ssp. lactis, was tested with a $2 \mu \mathrm{g}$ disc (Oxoid) by the diffusion method on both TSA and blood agar. Our isolate, however, was sensitive to clindamycin, forming a growth inhibition zone of 22 to $23 \mathrm{~mm}$ in diameter. While resistance to this antibiotic had been a constant feature in all freshwater and terrestrial isolates of $L$. garvieae available to us (trout from Turkey, Spain, England, France and Italy, catfish from Italy, and a bovine isolate from England), interestingly, the only other L. garvieae isolates that displayed sensitivity to clindamycin also had a marine origin (yellowtail from Japan). These findings question the sensitivity to clindamycin as a discriminative test between $L$. garvieae and $L$. lactis ssp. lactis.

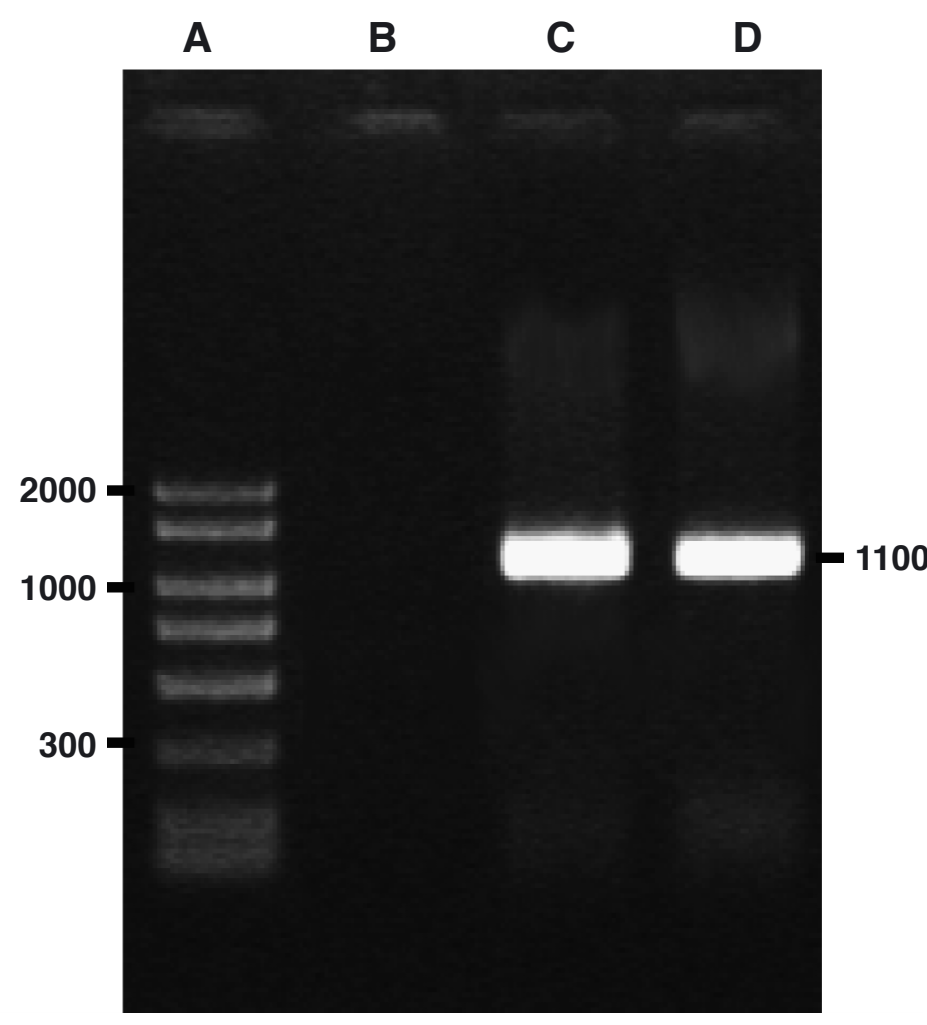

Fig. 1. Lactococcus garvieae PCR identification. Lanes: (A) AmpliSize ${ }^{\mathrm{TM}}$ molecular ruler (50 to 2000 bp ladder; BioRad); (B) negative control (no DNA); (C) Reference Strain NCDO 2155; (D) Israeli wrasse Coris aygula (present study). Numbers indicate size in bp of the marker (left) and the specific fragment (right)
Identification of our isolate as Lactococcus garvieae was confirmed by a PCR assay based on specific primers (pLG-1: 5'-CATAACAATGAGAATCGC-3' and pLG-2: 5'-GCACCCTCGCGG GTTG-3') deduced from the 16S rRNA gene (Zlotkin et al. 1998). PCR protocol was performed as described by Ravelo et al. (2003). The $1100 \mathrm{bp}$ amplification product was observed in both the Israeli isolate and the positive control (reference strain L. garvieae NCDO 2155), but not in the negative control (Fig. 1), supporting the results of the Rapid ID 32 Strep biochemical identification.

Two serotypes associated with the presence (serotype $\mathrm{KG}^{-}$) or absence (serotype $\mathrm{KG}^{+}$) of capsular material have been described within Lactococcus garvieae, with the capsule being associated with greater virulence (Romalde \& Toranzo 2002). Serological characterization, employing specific antisera against capsulated and non-capsulated strains of L. garvieae, demonstrated that the Israeli isolate belongs to the capsulated serotype $\mathrm{KG}^{-}$. The presence of a capsule was highlighted by the glutaraldehydelysine-fuchsin staining method (Ravelo et al. 2001b).

Random amplification of polymorphic DNA (RAPD) analysis with Primer 5 was used for molecular characterization of the isolate, as described by Ravelo et al. (2003). Representative strains of the 3 major genetic groups (Table 1) established by these authors were included for comparison, and showed the same profiles in this study. The Israeli isolate showed a unique profile (Fig. 2; Lane G), with less than $20 \%$ similarity with the other strains (Fig. 2; Lanes B to F) when the Dice coefficient (Dice 1945) was applied.

Several species of the genus Lactococcus are considered opportunistic pathogens with clinical significance for human and veterinary medicine, including L. garvieae, L. lactis ssp. lactis, and L. piscium (Eldar et al. 1996, Elliot \& Facklam 1996). L. garvieae has a worldwide distribution, is known to infect fish and shellfish in both freshwater and marine environments (Romalde \& Toranzo 2002), and has also been associated with mastitis in ruminants (Collins et al. 1983, Teixeira et al. 1996) and endocarditis in humans (Elliott et al. 1991, Fefer et al. 1998).

This is the first record of Lactococcus garvieae in the Red Sea. Recently, another pathogenic Grampositive coccus, Streptococcus iniae, was isolated from wild fishes of the Gulf of Eilat (Colorni et al. 2002). Although its origin could not be positively traced, local mariculture facilities were suspected as a key factor in the amplification and spreading of the infection. Similarly, cage farming in the Gulf of Eilat has been associated with the high prevalence of mycobacteriosis (by Mycobacterium marinum) found in wild rabbitfish Siganus rivulatus and other coastal 
fish species in the region (Diamant et al. 2000, Diamant 2001). Whether the present case of lactococcosis is a sporadic incident or is related to the deteriorating regional environmental quality remains to be determined, but the prevalence of certain diseases seems to have increased, as has the incidence of dead and moribund fish observed by divers in coastal waters, in circumstances similar to those in which the clown wrasse was found (Diamant 2001). Harvell et al. (1999) suggested that severe disease outbreaks in the wild are generated, in some cases, by a combination of global warming and human activities that have intensified international transport of marine species, thus bringing together pathogens and previously unexposed host populations. All these phenomena constitute an emerging trend affecting marine organisms world-wide.
The detection of 3 bacterial pathogens (Mycobacterium marinum, Streptococcus iniae and now Lactococcus garvieae) over a period of a few years in wild fish populations in the northern Red Sea is worrisome. While all 3 may be endemic Red Sea strains, comprehensive ichthyopathological studies, including bacteriological investigations, have been conducted in fish cultured in Eilat in the last 30 yr (Paperna et al. 1977, Colorni et al. 1981, Paperna 1983, 1991, Colorni 1992) and have failed to detect these disease agents, suggesting that either a surge in their virulence has occurred, or their appearance is the result of recent introduction into the region. Although all 3 are known to possess a zoonotic potential, human infections with these organisms are still unusual, as their pathogenicity probably depends on a combination of bacterial, host and environmental factors (Lehane \& Rawlin 2000).

Table 1. Lactococcus garvieae. Origin of the isolates used in this study. For sources, 'Laboratory collection' comes from Departamento de Microbiología y Parasitología, Universidad de Santiago (Spain); and NCDO is the National Collection of Dairy Organisms, Reading (UK). Genetic groups as described by Ravelo et al. (2003). na: not applicable

\begin{tabular}{|llccc|}
\hline Strain & \multicolumn{1}{c}{ Origin } & Year of isolation & Source & Genetic group \\
\hline Israeli wrasse & Red Sea wrasse, Israel & 2002 & Present study & na \\
TW 446.B3 & Rainbow trout, Spain & 1997 & Laboratory collection & A \\
$00-21$ & Rainbow trout, England & 2000 & Laboratory collection & A \\
B6 & Rainbow trout, Turkey & 2001 & Laboratory collection & A \\
3031 & Rainbow trout, France & 2001 & Laboratory collection & B \\
$309-$ C1 & Rainbow trout, Italy & 2000 & Laboratory collection & B \\
$297-8$ & Catfish, Italy & 1999 & Laboratory collection & A \\
YT-3 & Yellowtail, Japan & 1974 & Laboratory collection & C \\
NCDO 2155 & Cow, UK & 1973 & NCDO & C \\
\hline
\end{tabular}

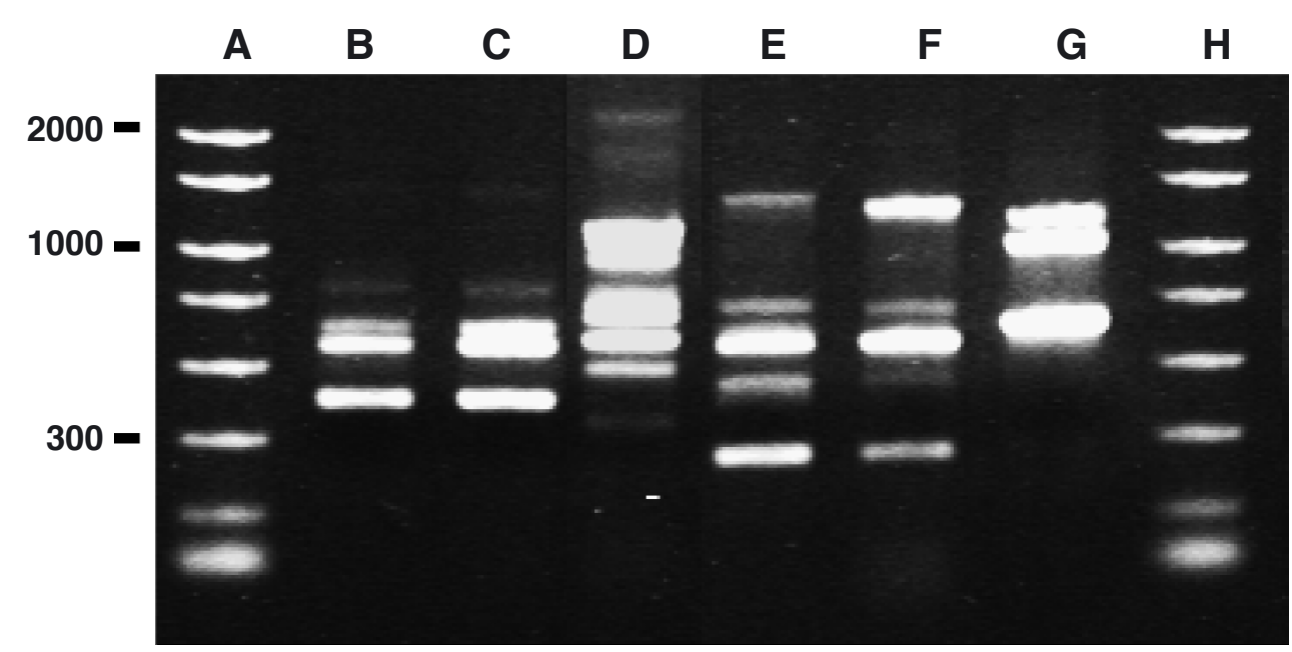

Fig. 2. Lactococcus garvieae RAPD fingerprints. Lanes: (A) and (H) AmpliSize ${ }^{\mathrm{TM}}$ molecular ruler (50 to 2000 bp ladder; Bio-Rad); (B) Strain TW446.B3; (C) Strain B6; (D) Strain 3031; (E) Strain YT-3; (F) Reference Strain NCDO 2155; (G) Israeli wrasse strain. Molecular sizes (in bp) are indicated on the left 
Acknowledgements. The technical assistance of Y. Shtupler and O. Carmi is gratefully acknowledged. This work was carried out at the Green-Keiser Fish Health Center, IOLR-NCM, Eilat, as part of IET Gulf of Eilat Monitoring and Research Program (2002-2003) funded by the Israeli Ministries of Agriculture, Environment and National Infrastructures. C.R., J.L.R. and A.E.T. were funded in part by Grant PTR19950471-OP from the Ministerio de Ciencia y Tecnología, Spain.

\section{LITERATURE CITED}

Collins MD, Farrow JA, Phillips BA, Kandler O (1983) Streptococcus garvieae sp. nov. and Streptococcus plantarum sp. nov. J Gen Microbiol 129:3427-3431

Colorni A (1992) A systemic mycobacteriosis in the European sea bass Dicentrarchus labrax cultured in Eilat (Red Sea). Isr J Aquacult Bamidgeh 44:75-81

Colorni A, Paperna I, Gordin H (1981) Bacterial infections in gilt-head sea bream Sparus aurata cultured at Elat. Aquaculture 23:257-267

Colorni A, Diamant A, Eldar A, Kvitt H, Zlotkin A (2002) Streptococcus iniae infections in Red Sea cage-cultured and wild fishes. Dis Aquat Org 49:165-170

Diamant A (2001) Cross-infections between marine cagecultured stocks and wild fish in the northern Red Sea: is the environment at risk? In: Rodgers CJ (ed) Proc OIE International Conference on Risk Analysis in Aquatic Animal Health. Office International des Epizooties, Paris, p 202-208

Diamant A, Banet A, Ucko M, Colorni A, Knibb W, Kvitt H (2000) Mycobacteriosis in wild rabbitfish Siganus rivulatus associated with cage farming in the Gulf of Eilat, Red Sea. Dis Aquat Org 39:211-219

Dice LR (1945) Measures of the amount of ecological association between species. Ecology 26:297-302

Eldar A, Ghittino C, Asanta L, Bozzetta E, Goria M, Prearo M, Bercovier H (1996) Enterococcus seriolicida is a junior synonym of Lactococcus garvieae, a causative agent of septicemia and meningoencephalitis in fish. Curr Microbiol 32:85-88

Elliott JA, Facklam RR (1996) Antimicrobial susceptibilities of Lactococcus lactis and Lactococcus garvieae as a proposed method to discriminate between them. J Clin Microbiol 34:1296-1298

Elliott JA, Collins MD, Pigott NE, Facklam RR (1991) Differentiation of Lactococcus lactis and Lactococcus garvieae from humans by comparison of whole-cell protein pat-

Editorial responsibility: Carey Cunningham, Aberdeen, UK terns. J Clin Microbiol 20:2731-2734

Fefer JJ, Ratzan KR, Sharp SE, Saiz E (1998) Lactococcus garvieae endocarditis: report of a case and review of the literature. Diagn Microbiol Infect Dis 32:127-130

Harvell CD, Kim K, Burkholder JM, Colwell RR and 9 others (1999) Emerging marine diseases-climate links and anthropogenic factors. Science 285:1505-1510

Lehane L, Rawlin GT (2000) Topically acquired bacterial zoonoses from fish: a review. Med J Aus 173:256-259

Paperna I (1983) Review of diseases of cultured warm-water marine fish. Rapp P-V Réun Cons Int Explor Mer 182:44-48

Paperna I (1991) Diseases caused by parasites in the aquaculture of warm water fish. Annu Rev Fish Dis 1:155-194

Paperna I, Colorni A, Gordin H, Kissil GW (1977) Diseases of Sparus aurata in marine culture at Elat. Aquaculture 10: 195-213

Randall JE (1983) Red sea fishes. IMMEL Publications, London

Ravelo C, Magariños B, Romalde JL, Toranzo AE (2001a) Conventional versus miniaturized systems for the phenotypic characterization of Lactococcus garvieae strains. Bull Eur Assoc Fish Pathol 21:136-144

Ravelo C, Magariños B, Toranzo AE, Romalde JL (2001b) Antigenic variation among Lactococcus garvieae strains and its relation with virulence. 10th Int Conf Eur Assoc Fish Pathol (EAFP), Dublin

Ravelo C, Magariños B, López-Romalde S, Toranzo AE, Romalde JL (2003) Molecular fingerprinting of fish pathogenic Lactococcus garvieae strains by RAPD analysis. J Clin Microbiol 41:751-756

Romalde JL, Toranzo AE (2002) Molecular approaches for the study and diagnosis of salmonid streptococcosis. In: Cunningham C (ed) Molecular diagnosis of salmonid diseases. Kluwer Academic, Dordrecht, p 211-233

Sheehan DC, Hrapchak BB (1980) Theory and practice of histotechnology, 2nd edn. CV Mosby Company, St Louis, $\mathrm{MO}$

Teixeira LM, Merquior VLC, Vianni MCE, Carvalho MGS, Fracalanzza SEL, Steigerwalt AG, Brenner DJ, Facklam RR (1996) Phenotypic and genotypic characterization of atypical Lactococcus garvieae strains isolated from water buffalos with subclinical mastitis and confirmation of $L$. garvieae as a senior subjective synonym of Enterococcus seriolicida. Int J Syst Bacteriol 46:664-668

Zlotkin A, Eldar A, Ghittino C, Bercovier H (1998) Identification of Lactococcus garvieae by PCR. J Clin Microbiol 36: 983-985

Submitted: March 24, 2003; Accepted: July 28, 2003 Proofs received from author(s): September 8, 2003 